Marianna Csörnyei*, Department of Mathematics, University College London, Gower Street, London WC1E 6BT, UK. e-mail: mari@math.ucl.ac.uk

Toby C. O'Neil, Department of Pure Mathematics, The Open University, Walton Hall, Milton Keynes, Bucks MK7 6AA, UK. e-mail:

t.c.oneil@open.ac.uk

David Preiss $\dagger$ Department of Mathematics, University College London, Gower Street, London WC1E 6BT, UK. e-mail: dp@math.ucl.ac.uk

\title{
THE COMPOSITION OF TWO DERIVATIVES HAS A FIXED POINT
}

\begin{abstract}
We show that if $f, g:[0,1] \rightarrow[0,1]$ are both Darboux Baire-1 functions, then their composition, $f \circ g$, possesses a fixed point.
\end{abstract}

In [3], Gibson and Natkaniec refer to a problem of K. C. Ciesielski who asked whether the composition of two derivative functions from the unit interval to the unit interval necessarily possesses a fixed point. A partial solution to this problem was given by P. Humke, R. Svetic and C. Weil in [4]. In this note we answer the question affirmatively. (An alternative proof has also been found by M. Elekes, T. Keleti and V. Prokaj, see [2]).

Recalling that a Baire-1 function is the pointwise limit of a sequence of continuous functions and that a Darboux function is one for which the image of any interval in its domain is connected, we can formulate our main result as follows.

Theorem 1. If $f, g:[0,1] \rightarrow[0,1]$ are both Darboux Baire-1 functions, then there is an $x \in[0,1]$ for which $(f \circ g)(x)=x$.

Since derivative functions are examples of Darboux Baire-1 functions, this answers Ciesielski's question. The rest of the paper consists of a proof of this theorem.

\footnotetext{
Key Words: Darboux, Baire-1, fixed points

Mathematical Reviews subject classification: 26A99, 26A21

Received by the editors September 18, 2000

*Partially supported by the Hungarian National Foundation for Scientific Research, grant \# F029768.

${ }^{\dagger}$ The authors wish to thank the Erdős Center for their support of the Summer School on Geometric and Dynamical Aspects of Measure Theory in Révfülöp during which this paper was written.
} 


\section{Proof of Theorem}

For $\phi: I \rightarrow \mathbf{R}, I$ an interval, we define

$$
\operatorname{graph}_{X}(\phi)=\{(x, \phi(x)): x \in I\}
$$

and

$$
\operatorname{graph}_{Y}(\phi)=\{(\phi(y), y): y \in I\}
$$

and given $a, b \in \mathbf{R}$, we let $[a, b],(a, b)$ denote the closed, and open intervals connecting them, respectively. For a point $p$ we let $(p)_{x}$ and $(p)_{y}$ denote the $x$ and $y$ coordinates of $p$, respectively.

Fix two Darboux Baire-1 functions $f, g:[0,1] \rightarrow[0,1]$. Set

$$
F=\operatorname{graph}_{X}(f)=\left\{(x, f(x)) \in[0,1]^{2}: x \in[0,1]\right\}
$$

and

$$
G=\operatorname{graph}_{Y}(g)=\left\{(g(y), y) \in[0,1]^{2}: y \in[0,1]\right\},
$$

then in order to prove the theorem it is sufficient to show that $F \cap G \neq \emptyset$. We may assume without loss of generality that

$$
f(0)=0, f(1)=1
$$

and

$$
g(0)=1, g(1)=0
$$

for, by considering the square $[-1,2] \times[-1,2]$ and extending the sets $F$ and $G$ as indicated in Figure 1, and then rescaling, we can define two new Darboux Baire-1 functions $\tilde{f}, \tilde{g}:[0,1] \rightarrow[0,1]$ with $\tilde{f}(0)=0, \tilde{f}(1)=1, \tilde{g}(0)=1$ and $\tilde{g}(1)=0$ whose composition possesses a fixed point if and only if the original functions did.

Throughout this note, by rectangle we understand a rectangle whose sides are parallel to the usual coordinate axes. Topological notions like open, closed, etc., will be considered relatively to $[0,1]^{2}$.

Definition 1. We define a crossing-configuration, $\mathcal{R}=(A, B)$ to be an ordered pair consisting of non-empty finite subsets $A$ and $B$ of $F$ and $G$, respectively, such that whenever $I$ and $J$ are closed intervals with $A \cup B \subset I \times J$ and $\phi: I \rightarrow \mathbf{R}$ and $\psi: J \rightarrow \mathbf{R}$ are continuous functions with:

$$
\begin{gathered}
A \subset \operatorname{graph}_{X}(\phi) \quad \text { and } \\
B \subset \operatorname{graph}_{Y}(\psi),
\end{gathered}
$$

then

$$
\operatorname{graph}_{X}(\phi) \cap \operatorname{graph}_{Y}(\psi) \neq \emptyset .
$$




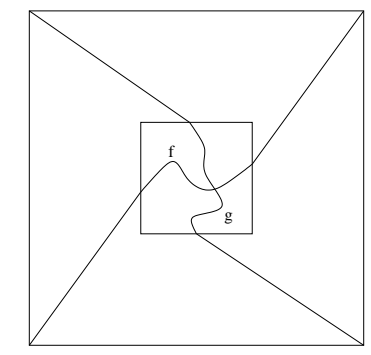

Figure 1: Ensuring that $f(0)=0, f(1)=1$ and $g(0)=1, g(1)=0$

Remark 1. If $\mathcal{R}=(A, B)$ is a crossing configuration, and if $\phi, \psi: I, J \rightarrow$ $[0,1]$ are continuous functions satisfying (1) and (2) respectively, then for any rectangle $R \subset[0,1]^{2}$ which contains $A \cup B$, we know that

$$
\operatorname{graph}_{X}(\phi) \cap \operatorname{graph}_{Y}(\psi) \cap R \neq \emptyset .
$$

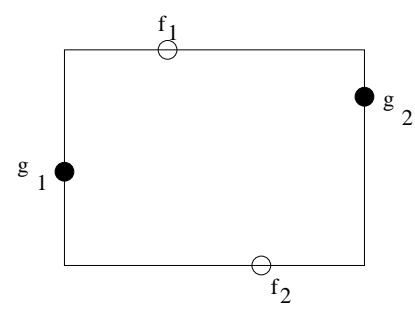

(a)

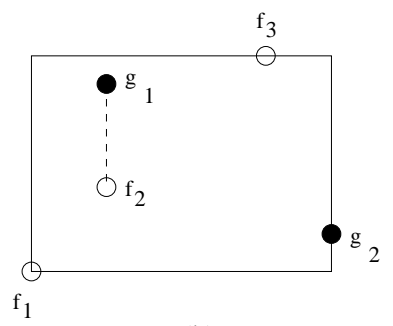

(b)

Figure 2: Two examples of crossing configurations: points denoted by $\circ$ lie in $F$ and points denoted by $\bullet$ lie in $G$.

Lemma 1. The configurations illustrated in Figure 2 are crossing configurations.

Proof. Figure 2(a): Here $f_{1}, f_{2}$ are points from $F$ lying on the top and bottom edges of a closed rectangle, and $g_{1}, g_{2}$ are points of $G$ lying on the left and right edges of the rectangle, respectively. Suppose that $\phi$ and $\psi$ are continuous functions with $\left\{f_{1}, f_{2}\right\} \subset \operatorname{graph}_{X}(\phi)$ and $\left\{g_{1}, g_{2}\right\} \subset \operatorname{graph}_{Y}(\psi)$. Notice that the part of graph ${ }_{X}(\phi)$ lying within the vertical strip whose edges 
contain $f_{1}$ and $f_{2}$ may be extended to form a Jordan curve separating $g_{1}$ and $g_{2}$ in such a way that the added curve does not intersect $\operatorname{graph}_{Y}(\psi) \cap(\mathbf{R} \times$ $\left.\left[\left(g_{1}\right)_{y},\left(g_{2}\right)_{y}\right]\right)$. (See Figure 3.) that

Since $\operatorname{graph}_{Y}(\psi) \cap\left(\mathbf{R} \times\left[\left(g_{1}\right)_{y},\left(g_{2}\right)_{y}\right]\right)$ connects $g_{1}$ with $g_{2}$, we conclude

$$
\left(\operatorname{graph}_{Y}(\psi) \cap\left(\mathbf{R} \times\left[\left(g_{1}\right)_{y},\left(g_{2}\right)_{y}\right]\right)\right) \cap\left(\operatorname{graph}_{X}(\phi) \cap\left(\left[\left(f_{1}\right)_{x},\left(f_{2}\right)_{x}\right] \times \mathbf{R}\right)\right) \neq \emptyset,
$$

as required.

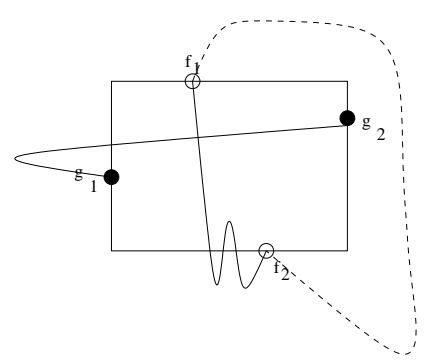

Figure 3: $\operatorname{graph}_{X}(\phi) \cap\left(\left[\left(f_{1}\right)_{x},\left(f_{2}\right)_{x}\right] \times \mathbf{R}\right)$ may be extended to form a Jordan curve separating $g_{1}$ and $g_{2}$.

Figure 2(b): In this situation we have three points $f_{1}, f_{2}, f_{3} \in F$ and two points $g_{1}, g_{2} \in G$ with $\left(f_{1}\right)_{x}<\left(f_{2}\right)_{x}=\left(g_{1}\right)_{x}<\left(g_{2}\right)_{x},\left(f_{1}\right)_{y} \leq\left(f_{2}\right)_{y} \leq$ $\left(g_{1}\right)_{y} \leq\left(f_{3}\right)_{y}$ and $\left(f_{1}\right)_{y} \leq\left(g_{2}\right)_{y} \leq\left(f_{3}\right)_{y}$. Suppose that $\phi$ and $\psi$ are continuous functions with $\left\{f_{1}, f_{2}, f_{3}\right\} \subset \operatorname{graph}_{X}(\phi)$ and $\left\{g_{1}, g_{2}\right\} \subset \operatorname{graph}_{Y}(\psi)$. Observe that the part of $\operatorname{graph}_{X}(\phi)$ lying within the vertical strip whose edges contain $f_{1}$ and $f_{3}$ may be extended to form a Jordan curve separating $g_{1}$ and $g_{2}$ in such a way that the added curve does not intersect $\operatorname{graph}_{Y}(\phi) \cap\left(\mathbf{R} \times\left[\left(g_{1}\right)_{y},\left(g_{2}\right)_{y}\right]\right)$. (See Figure 4.)

Since $\operatorname{graph}_{Y}(\psi) \cap\left(\mathbf{R} \times\left[\left(g_{1}\right)_{y},\left(g_{2}\right)_{y}\right]\right)$ connects $g_{1}$ and $g_{2}$, we conclude that

$$
\left(\operatorname{graph}_{Y}(\psi) \cap\left(\mathbf{R} \times\left[\left(g_{1}\right)_{y},\left(g_{2}\right)_{y}\right]\right)\right) \cap\left(\operatorname{graph}_{X}(\phi) \cap\left(\left[\left(f_{1}\right)_{x},\left(f_{2}\right)_{x}\right] \times \mathbf{R}\right)\right) \neq \emptyset,
$$

as required.

Remark 2. Since $(0,0)$ and $(1,1) \in F$, and $(0,1),(1,0) \in G$, we conclude that $\mathcal{R}_{0}=(\{(0,0),(1,1)\},\{(0,1),(1,0)\})$ is a crossing configuration.

The key part of our argument is the following proposition. 


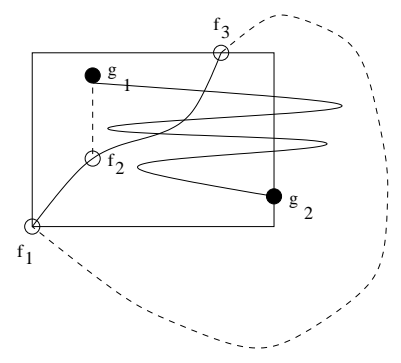

Figure 4: $\operatorname{graph}_{X}(\phi) \cap\left(\left[\left(f_{1}\right)_{x},\left(f_{3}\right)_{x}\right] \times \mathbf{R}\right)$ may be extended to form a Jordan curve separating $g_{1}$ and $g_{2}$.

Proposition 2. For all crossing-configurations $\mathcal{R}=(A, B)$ and for all open rectangles $R \supset A \cup B$ and open sets $U \supset F$ (or $V \supset G$ ), we can find a crossingconfiguration $\mathcal{R}^{\prime}=\left(A^{\prime}, B^{\prime}\right)$ and a closed rectangle $R^{\prime}$ with $A^{\prime} \cup B^{\prime} \subset R^{\prime} \subset U \cap R$ (or $V \cap R$ ).

Before proving this, we show how it immediately leads to a proof of Theorem 1: Since $f$ and $g$ are Darboux, Baire-1 functions, their graphs are $G_{\delta}$ subsets of the plane (see [5, Ch.II.§31, VII, Thm 1]), so

$$
F=\bigcap_{n=1}^{\infty} U_{n}, \quad \text { where } U_{1} \supset U_{2} \supset \cdots \text { are open sets }
$$

and

$$
G=\bigcap_{n=1}^{\infty} V_{n}, \quad \text { where } V_{1} \supset V_{2} \supset \cdots \text { are open sets. }
$$

We recall, from Remark 2, that

$$
\mathcal{R}_{0}=(\{(0,0),(1,1)\},\{(0,1),(1,0)\})
$$

is a crossing-configuration. We now use the proposition to find a sequence of crossing-configurations $\mathcal{R}_{i}=\left(A_{i}, B_{i}\right)$ and open rectangles $R_{i} \supset A_{i} \cup B_{i}$ such that

$$
\operatorname{cl}\left(R_{2 i+1}\right) \subset R_{2 i} \cap U_{i+1}
$$

and

$$
\operatorname{cl}\left(R_{2(i+1)}\right) \subset R_{2 i+1} \cap V_{i+1}
$$

for $i=0,1,2, \ldots$. Hence

$$
\operatorname{cl}\left(R_{0}\right)=R_{0} \supset \operatorname{cl}\left(R_{1}\right) \supset R_{2} \supset \operatorname{cl}\left(R_{3}\right) \supset \cdots,
$$




$$
\emptyset \neq \bigcap_{n=1}^{\infty} \operatorname{cl}\left(R_{n}\right) \subset \bigcap_{n} U_{n}=F
$$

and

$$
\emptyset \neq \bigcap_{n=1}^{\infty} \operatorname{cl}\left(R_{n}\right) \subset \bigcap_{n} V_{n}=G,
$$

which together imply that $F \cap G \neq \emptyset$ as required.

Proof. We prove the proposition 2 for the case when $U \supset F$, the case when $V \supset G$ is similar.

Suppose that $\mathcal{R}=(A, B)$ is a crossing configuration, $R \supset A \cup B$ is an open rectangle, and let $S=\left[x_{1}, x_{2}\right] \times\left[y_{1}, y_{2}\right] \subset R$ be a closed rectangle whose (relative) interior contains $A \cup B$.

Observe that if $\phi:\left[x_{1}, x_{2}\right] \rightarrow \mathbf{R}$ were a continuous function for which $A \subset$ $\operatorname{graph}_{X}(\phi)$ and $\operatorname{graph}_{X}(\phi) \cap G \cap S=\emptyset$, then $G \subset[0,1]^{2} \backslash\left(\operatorname{graph}_{X}(\phi) \cap S\right)$ would be a relatively open set. Since $g \in D B_{1}$, it is almost continuous [1], so there exists a continuous function $\psi:[0,1] \rightarrow \mathbf{R}$ with $\operatorname{graph}_{Y}(\psi) \subset \mathbf{R} \times[0,1] \backslash$ $\left(\operatorname{graph}_{X}(\phi) \cap S\right)$. Moreover, we can require that $B \subset \operatorname{graph}_{Y}(\psi)$. (See e.g. [6, Lemma 6.2].) Hence $\operatorname{graph}_{X}(\phi) \cap \operatorname{graph}_{Y}(\psi) \cap S=\emptyset$. But $(A, B)$ is a crossing configuration - a contradiction.

We suppose that there are no crossing-configurations $\left(A^{\prime}, B^{\prime}\right)$ and closed rectangles $R^{\prime}$ with

$$
A^{\prime} \cup B^{\prime} \subset R^{\prime} \subset U \cap R
$$

(noting that this implies $F \cap G \cap R=\emptyset$ ). We will prove that this implies that there is a continuous function $\phi:\left[x_{1}, x_{2}\right] \rightarrow \mathbf{R}$ with $A \subset \operatorname{graph}_{X}(\phi)$ and $\operatorname{graph}_{X}(\phi) \cap G \cap S=\emptyset$ giving us our required contradiction.

We do this via the method of regular intervals: we say an interval $I \subset$ $\left[x_{1}, x_{2}\right]$ is regular, if for all $s, t \in I, s<t$, we can find a continuous function $\phi:[s, t] \rightarrow \mathbf{R}$ for which

$$
\phi(s)=f(s), \quad \phi(t)=f(t)
$$

and

$$
\operatorname{graph}_{X}(\phi) \cap G \cap S=\emptyset .
$$

(Note that regular intervals need neither be open nor closed.) If we show that $\left[x_{1}, x_{2}\right]$ is itself regular, then we are done.

It is easy to see that:

(1) If $I$ and $J$ are regular intervals and $I \cap J \neq \emptyset$, then $I \cup J$ is regular;

(2) If $I$ is an interval which is the (finite or infinite) union of open regular intervals, then $I$ is regular. 
It is slightly trickier to verify:

(3) If $I$ is regular, then $\operatorname{cl}(I)$ is regular.

Proof of (3): Let $r$ be the left endpoint of $I$. (The proof for the right endpoint is similar.) It is enough to show that we can find $r^{\prime}>r$ arbitrarily close to $r$ for which there is a continuous function $\phi:\left[r, r^{\prime}\right] \rightarrow \mathbf{R}$ such that $\phi(r)=f(r)$, $\phi\left(r^{\prime}\right)=f\left(r^{\prime}\right)$ and $\operatorname{graph}_{X}(\phi) \cap G \cap S=\emptyset$.

Choose $r_{i} \in I$ such that $r_{1}>r_{2}>\cdots>r, r_{n} \rightarrow r$ and for which $f\left(r_{n}\right) \rightarrow$ $f(r)$ (the Darboux property for $f$ ensures we can find such a sequence). For each $k \in \mathbf{N}$, we can find $n_{k}$ such that

$$
f\left(r_{n_{k}}\right) \in\left(f(r)-2^{-k}, f(r)+2^{-k}\right)
$$

and both

$$
g\left(f(r)-2^{-k}\right) \text { and } g\left(f(r)+2^{-k}\right) \notin\left(r, r_{n_{k}}\right) .
$$

Fix a sequence $n_{1}<n_{2}<n_{3}<\cdots$ with this property. Since $I$ is regular we can find a sequence of continuous functions $\phi_{k}:\left[r_{n_{k+1}}, r_{n_{k}}\right] \rightarrow \mathbf{R}$ for which

$$
\phi_{k}\left(r_{n_{k}}\right)=f\left(r_{n_{k}}\right), \phi_{k}\left(r_{n_{k+1}}\right)=f\left(r_{n_{k+1}}\right) \text { and } \operatorname{graph}_{X}\left(\phi_{k}\right) \cap G \cap S=\emptyset .
$$

Then the function $\tilde{\phi}:\left[r, r_{n_{1}}\right] \rightarrow \mathbf{R}$ defined by

$$
\tilde{\phi}(x)= \begin{cases}f(r) & \text { if } x=r \\ \max \left\{\min \left\{\phi_{k}(x), f(r)+2^{-k}\right\}, f(r)-2^{-k}\right\} & \text { if } x \in\left[r_{n_{k+1}}, r_{n_{k}}\right]\end{cases}
$$

is a well-defined continuous function for which $\tilde{\phi}\left(r_{n_{k}}\right)=f\left(r_{n_{k}}\right)$ for all $k$, $\tilde{\phi}(r)=f(r)$ and $\operatorname{graph}_{X}(\tilde{\phi}) \cap G \cap S=\emptyset$.

Suppose that $\left[x_{1}, x_{2}\right]$ is not a regular interval and let

$$
P=\left[x_{1}, x_{2}\right] \backslash \bigcup\left\{I \subset\left[x_{1}, x_{2}\right]: I \text { is relatively open and regular }\right\} .
$$

Then $P$ is closed, and observations (2) and (3) imply that $P \cap\left(x_{1}, x_{2}\right)$ is nonempty and has no isolated points. Since $f$ is a Baire- 1 function, the continuity points of $\left.f\right|_{P}$ form a dense $G_{\delta}$ set in $P$ [5, Ch.II.§31, X, Thm 1]. Thus we can choose $r \in P \cap\left(x_{1}, x_{2}\right)$ such that

- $\left.f\right|_{P}$ is continuous at $r$; and

- $r$ is not the endpoint of any interval in $\left[x_{1}, x_{2}\right] \backslash P$ which is contiguous to $P$. 
Without loss of generality we can assume that $g(f(r))>r$. We will show that in this case we can always find $r^{\prime}>r$ for which $\left(r, r^{\prime}\right)$ is regular which contradicts our choice of $r$.

Since the endpoints of any interval contiguous to $P$ belong to $P$, and the closure of any regular interval is also regular, then by (1) it is enough to find an $r^{\prime}>r$ such that for $s, t \in\left(r, r^{\prime}\right) \cap P$ we can find continuous $\phi:[s, t] \rightarrow \mathbf{R}$ with $\phi(s)=f(s), \phi(t)=f(t)$ and $\operatorname{graph}_{X}(\phi) \cap G \cap S=\emptyset$. We can assume that there is no $r^{\prime}>r$ for which $\left.f\right|_{\left(r, r^{\prime}\right)}$ is constant.

Case 1: $(r, f(r)) \notin S$.

In this case, since $\left.f\right|_{P}$ is continuous at $r$, the existence of $r^{\prime}$ is trivial.

Case 2: $(r, f(r)) \in S$ and there is no $r^{*}>r$ for which either $\left.f\right|_{\left(r, r^{*}\right)} \geq f(r)$ or $\left.f\right|_{\left(r, r^{*}\right)} \leq f(r)$.

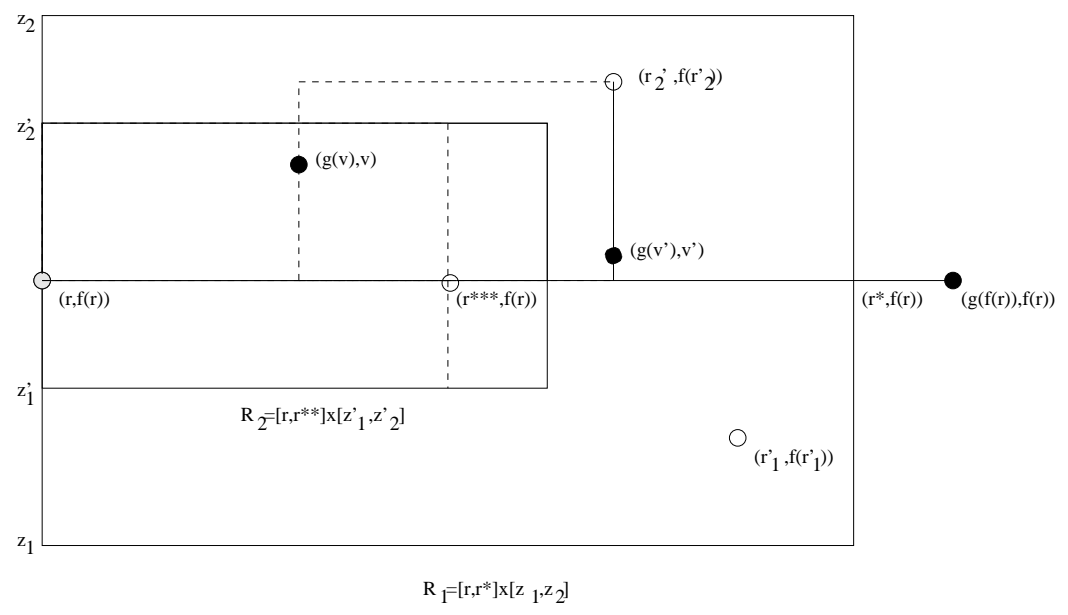

Figure 5: Case 2

Choose $r<r^{*}<g(f(r))$ and $z_{1}<f(r)<z_{2}$ for which $R_{1}=\left[r, r^{*}\right] \times$ $\left[z_{1}, z_{2}\right] \subset R \cap U$. (See Figure 5.) Then we can find $r_{1}^{\prime}, r_{2}^{\prime} \in\left(r, r^{*}\right)$ such that

$$
z_{1}<f\left(r_{1}^{\prime}\right)<f(r)<f\left(r_{2}^{\prime}\right)<z_{2} .
$$

Now we choose $z_{1}^{\prime}, z_{2}^{\prime}$ and $r<r^{* *}<\min \left\{r_{1}^{\prime}, r_{2}^{\prime}\right\}$ such that

$$
\begin{gathered}
f\left(r_{1}^{\prime}\right)<z_{1}^{\prime}<f(r)<z_{2}^{\prime}<f\left(r_{2}^{\prime}\right), \\
R_{2}=\left[r, r^{* *}\right] \times\left[z_{1}^{\prime}, z_{2}^{\prime}\right] \subset R_{1} \quad \text { and }
\end{gathered}
$$




$$
\operatorname{graph}_{X}\left(\left.f\right|_{P \cap\left[r, r^{* *}\right]}\right) \subset R_{2} .
$$

Finally by the Darboux property, we can find $r^{* * *} \in\left(r, r^{* *}\right)$ for which $f\left(r^{* * *}\right)=f(r)$.

Claim: $\left(\left[r, r^{* * *}\right] \times\left[z_{1}^{\prime}, z_{2}^{\prime}\right]\right) \cap G=\emptyset$.

Proof of claim: For suppose $(g(v), v) \in\left[r, r^{* * *}\right] \times\left[z_{1}^{\prime}, z_{2}^{\prime}\right]$, without loss of generality we can assume that $v>f(r)$. By the Darboux property applied to $g$ we can find $v^{\prime} \in[f(r), v]$ with $g\left(v^{\prime}\right)=r_{2}^{\prime}$. But then

$$
\left(\left\{\left(r^{* * *}, f\left(r^{* * *}\right)\right),\left(r_{2}^{\prime}, f\left(r_{2}^{\prime}\right)\right)\right\},\left\{(g(v), v),\left(g\left(v^{\prime}\right), v^{\prime}\right)\right\}\right)
$$

is a crossing-configuration of the type (a) illustrated in Figure 2 contained in

$$
\left[g(v), g\left(v^{\prime}\right)\right] \times\left[f(r), f\left(r_{2}^{\prime}\right)\right] \subset R \cap U,
$$

which is a contradiction.

But now clearly the interval $\left(r, r^{* * *}\right)$ is regular.

Case 3: $(r, f(r)) \in S$ and there is $r^{*}>r$ such that $\left.f\right|_{\left(r, r^{*}\right)} \geq f(r)$. (Or $(r, f(r)) \in S$ and there is $r^{*}>r$ such that $\left.f\right|_{\left(r, r^{*}\right)} \leq f(r)$.)

Without loss of generality, we do the case when there is an $r^{*}>r$ with $\left.f\right|_{\left(r, r^{*}\right)} \geq f(r)$ and there is no $r^{\prime}>r$ for which $\left.f\right|_{\left(r, r^{\prime}\right)}$ is constant. Choose $z_{2}>f(r)$ and $r^{*}>r$ for which $\left.f\right|_{\left(r, r^{*}\right)} \geq f(r)$ and $\left[r, r^{*}\right] \times\left[f(r), z_{2}\right] \subset R \cap U$ and set $R_{1}=\left[r, r^{*}\right] \times\left[f(r), z_{2}\right]$.

Since $\left.f\right|_{\left(r, r^{*}\right)}$ is not constant (and so $f(r)<1$ ), then we can find $r<r_{2}^{\prime}<r^{*}$ such that $f(r)<f\left(r_{2}^{\prime}\right)<z_{2}$. Choose $R_{2}=\left[r, r^{\prime \prime}\right] \times\left[f(r), z_{2}^{\prime}\right]$ such that

$$
\begin{gathered}
f(r)<z_{2}^{\prime}<f\left(r_{2}^{\prime}\right), \quad r<r^{\prime \prime}<r_{2}^{\prime} \quad \text { and } \\
\operatorname{graph}_{X}\left(\left.f\right|_{P \cap\left[r, r^{\prime \prime}\right]}\right) \subset R_{2} .
\end{gathered}
$$

We show that there are no points $(u, f(u)),(g(v), v)$ in $R_{2}$ for which $u=g(v)$ and $f(u)<v$. For if there were, we could use the fact that $g$ is Darboux to find $w \in(f(r), v)$ for which $g(w)=r_{2}^{\prime}$ and then we would have a crossingconfiguration, namely $\left(\left\{(u, f(u)),(r, f(r)),\left(r_{2}^{\prime}, f\left(r_{2}^{\prime}\right)\right)\right\},\{(g(v), v),(g(w), w)\}\right)$ contained in $R_{1}$, see Lemma 1 and Figure 6.

Lemma 2. There is a rectangle $R_{3}=\left[r, r^{\prime \prime \prime}\right] \times\left[f(r), z_{2}^{\prime \prime}\right] \subset R_{2}$ such that $\operatorname{graph}_{X}\left(\left.f\right|_{P \cap\left[r, r^{\prime \prime \prime}\right]}\right) \subset R_{3}$ and there are no points $(u, f(u)),(g(v), v) \in R_{3}$ for which $g(v) \leq u, f(u) \leq v$.

Proof. If $R_{3}=R_{2}$ does not satisfy the lemma, then there is $\left(u_{0}, f\left(u_{0}\right)\right)$ and $\left(g\left(v_{0}\right), v_{0}\right) \in R_{2}$ for which

$$
g\left(v_{0}\right) \leq u_{0} \quad \text { and } \quad f\left(u_{0}\right) \leq v_{0} .
$$




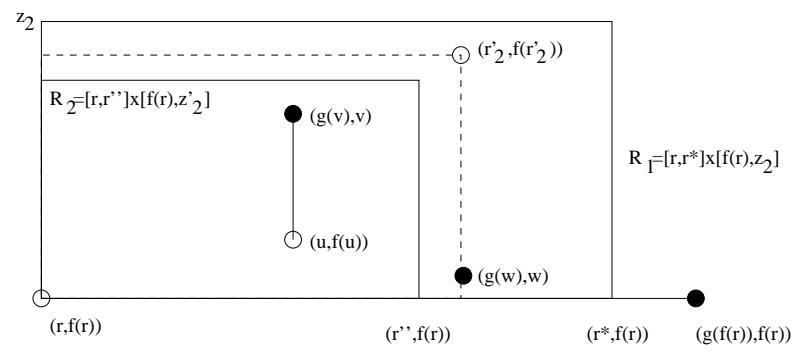

Figure 6: Diagram illustrating occurrence of a crossing-configuration in Case 3 if we can find $u=g(v)$ and $f(u)<v$ in $R_{2}$.

Choose $f(r)<z_{2}^{\prime \prime}<f\left(u_{0}\right)$ and use the fact that $r$ is a continuity point of $\left.f\right|_{P}$ to find $r<r^{\prime \prime \prime}<g\left(v_{0}\right)$ for which $\operatorname{graph}_{X}\left(\left.f\right|_{P \cap\left[r, r^{\prime \prime \prime}\right]}\right) \subset R_{3}=\left[r, r^{\prime \prime \prime}\right] \times\left[f(r), z_{2}^{\prime \prime}\right]$.

Suppose now that we can find $(u, f(u))$ and $(g(v), v)$ in $R_{3}$ for which $g(v) \leq$ $u$ and $f(u) \leq v$. Then by the Darboux property for $g$, we can find a point $\left(g\left(v_{2}\right), v_{2}\right)$ with $v_{2} \in\left(v, v_{0}\right)$ and $g\left(v_{2}\right)=f(u)$ but then $u=g\left(v_{2}\right)$ and $f(u)<v_{2}$ contradicting the observation made just before this lemma; see Figure 7 . Hence the lemma holds.

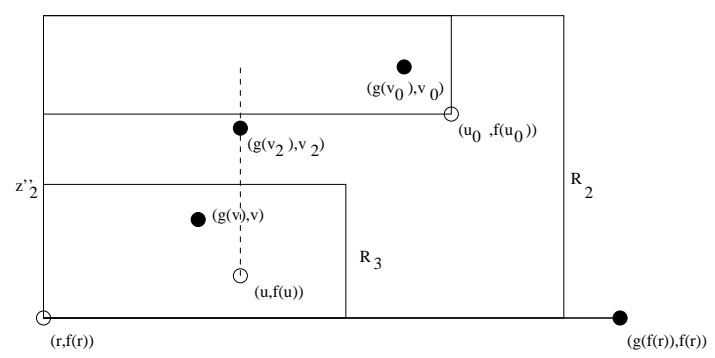

Figure 7: The figure for Lemma 2

We now prove that the interval $\left(r, r^{\prime \prime \prime}\right)$ we have constructed is regular; that is, for all $s, t \in P \cap\left[r, r^{\prime \prime \prime}\right]$ with $s<t$, we can find a continuous function $\phi:[s, t] \rightarrow \mathbf{R}$ for which graph ${ }_{X}(\phi) \cap G \cap S=\emptyset$, and $\phi(s)=f(s)$ and $\phi(t)=f(t)$. We know that $(s, f(s))$ and $(t, f(t))$ are in $R_{3}$. We can assume that $s$ is not the left endpoint of an interval contiguous to $P$. If $f(s) \geq f(t)$, then Lemma 2 allows us to choose $\phi$ to be the affine function joining $f(s)$ and $f(t)$. If $f(s)<f(t)$ we distinguish two cases: 
(A): There is $u \in(s, t)$ such that $f(u) \leq f(s)$.

In this case, Lemma 2 implies that $G$ does not meet the shaded region of Figure 8 and we can join $(s, f(s),(u, f(t))$ and $(t, f(t))$ by a piecewise linear function.

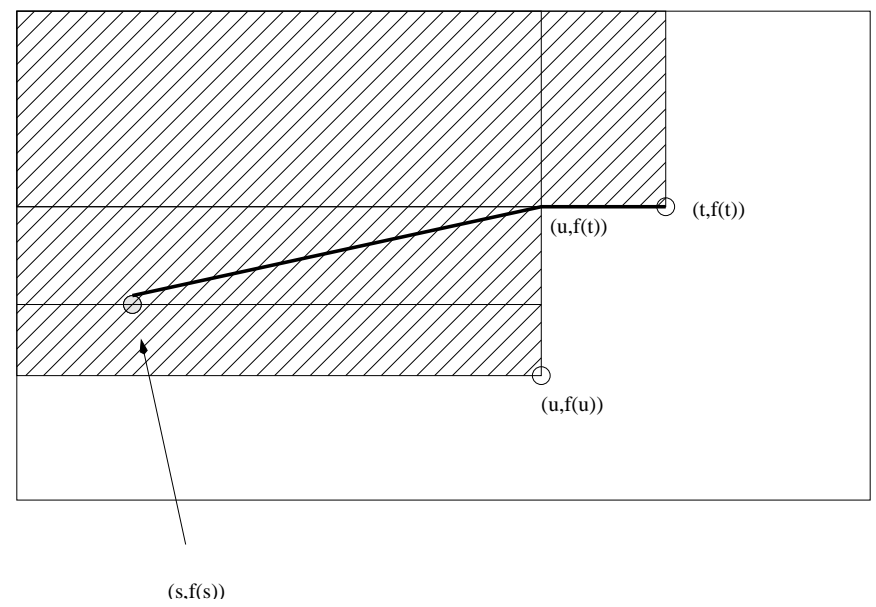

Figure 8: Constructing our continuous function $\phi$ in Case (A).

(B): There is a sequence $\left(u_{n}\right)$ with $t>u_{1}>u_{2}>\cdots \rightarrow s$ such that $f(t)>$ $f\left(u_{1}\right)>f\left(u_{2}\right)>\cdots \rightarrow f(s)$. In this case we can join the points $(t, f(t))$, $\left(u_{1}, f(t)\right),\left(u_{2}, f\left(u_{1}\right)\right),\left(u_{3}, f\left(u_{2}\right)\right), \ldots$ piecewise linearly, see Figure 9.

\section{Open problems}

There are a couple of natural questions suggested by this result:

1. is the graph of the composition of two Darboux Baire-1 functions connected?

2. does a similar result hold for the composition of $n$ Darboux Baire-1 functions when $n \geq 3$ ?

\section{References}

[1] J.B. Brown, Almost continuous Darboux functions and Reed's pointwise convergence criteria, Fundam. Math., 86(1974), 1-7. 


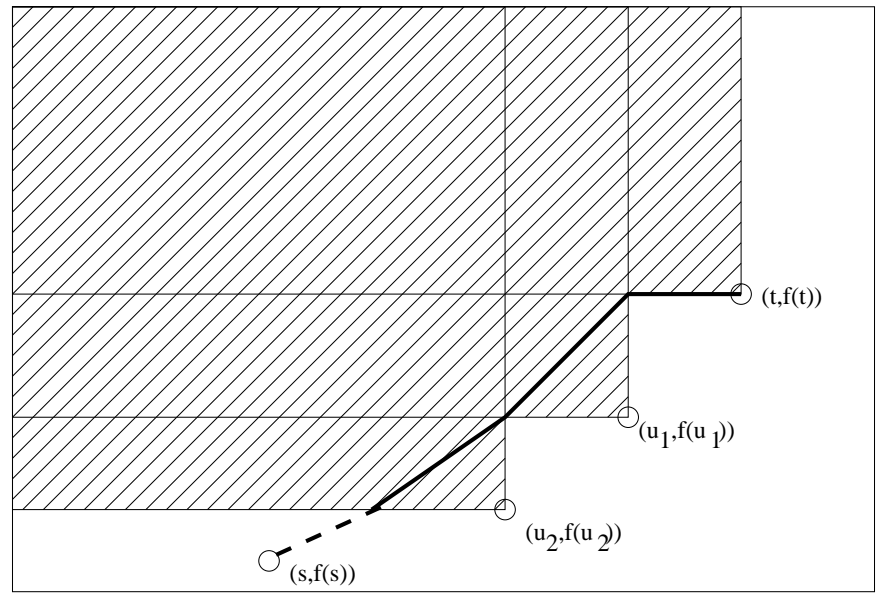

Figure 9: Constructing our continuous function $\phi$ in Case (B).

[2] M. Elekes, T. Keleti, V. Prokaj, The Fixed Point of the Composition of Derivatives, submitted to Real Analysis Exch.

[3] R. G. Gibson and T. Natkaniec, Darboux Like Functions. Old Problems and New Results, Real Analysis Exch., 24(1998/9), 487-496.

[4] P. Humke, R. Svetic and C. Weil, A Darboux Baire One Fixed Point Problem, to appear in Real Analysis Exch.

[5] K. Kuratowski, Topology I, 1966, Academic Press.

[6] T. Natkaniec, Almost continuity, Real Analysis Exch., 17(1991/2), 462520. 